\title{
Identifying and Managing Sources of Variability in Cell Therapy Manufacturing and Clinical Trials
}

\author{
Lara lonescu Silverman $^{1,2}$ (D) Flagg Flanagan ${ }^{1} \cdot$ Daniel Rodriguez-Granrose ${ }^{1,3} \cdot$ Katie Simpson $^{1} \cdot$ Lindsey Hart Saxon $^{1}$. \\ Kevin T. Foley ${ }^{1,2,4}$
}

Received: 25 July 2019 / Revised: 25 July 2019 / Accepted: 22 August 2019/Published online: 5 September 2019

(C) The Author(s) 2019

\begin{abstract}
Identifying and managing cell therapy variability can be a significant challenge for a company seeking to commercialize a new product. Failure to address this issue can lead to negative consequences such as delayed approval due to unsuccessful clinical investigations, or failed product lots that do not meet release criteria. Allogeneic cell therapies can be particularly prone to variability challenges due to the use of variable input material. In order to support the manufacturers of cell therapies, the FDA has identified two primary regulatory pathways (351 vs 361) that reflect the relative risk of the product. In this review, we will discuss criteria that separate the two potential regulatory pathways for cell therapy products in the USA. Also, we will discuss what aspects of manufacturing and clinical trial execution might introduce undesired variability that can derail the path towards licensure and commercialization, along with tools to minimize these potential sources of variability. ClinicalTrials.gov Identifier: NCT03347708 and NCT03955315

\section{Lay Summary}

Therapies that utilize live cells as the active ingredient, known as cell therapies, are a promising approach to treating many diseases that cannot be addressed with traditional medicines. However, with this great potential comes specific challenges associated with cell therapy, including identifying the appropriate regulatory approval pathway, manufacturing it in a reproducible way, and successfully executing clinical trials. In this review, we describe potential sources of variability that can negatively impact the translation of a cell therapy, and ways to minimize those risks.
\end{abstract}

Keywords Cell therapy $\cdot$ Regenerative medicine $\cdot$ Manufacturing $\cdot$ Clinical trials

\section{Introduction}

Cellular therapies are an extremely promising medical approach to treating many previously intractable diseases. The unique mechanisms of action available when a living cell serves as

Lara Ionescu Silverman

lara@discgenics.com

1 DiscGenics, Inc., 5940 Harold Gatty Drive, Salt Lake City, UT 84116, USA

2 Department of Neurosurgery, University of Tennessee Health Science Center, 910 Madison Ave, Memphis, TN, 38163, USA

3 Department of Biochemistry and Molecular Biology, University of Miami, 1011 NW 15th St, Miami, FL 33136, USA

4 Semmes-Murphey Clinic, 6325 Humphreys Blvd, Memphis, TN 38120, USA the active ingredient allow for these therapies to address important unmet clinical needs. These treatments have the potential to revolutionize healthcare and make significant strides in helping patients. The FDA has identified multiple pathways to facilitate patient access to such products depending on the risk posed to the patient. While some pathways are shorter and require less rigor, products allowed on these pathways have limited ability to make claims of clinical efficacy, given the lack of manufacturing and clinical data. For more complex cellular therapies that require close FDA oversight, as of July 2019, 17 products have been approved in the USA for commercial use [1]. According to the Alliance for Regenerative Medicine, an industry organization that supports the cell therapy field, in Q1 of 2019, there were 1060 active clinical studies in the field of regenerative medicine, 642 of which were for cell therapies or gene-modified cell therapies [2]. In recent years, the field of cell therapy has seen significant milestones with two major approvals that have tremendous market potential, including 
KYMRIAH and YESCARTA. In response to the recent approvals as well as the rich clinical pipeline of potential products, the regulatory pathway is evolving to accommodate this fastmoving field.

While the clinical prospects are promising, taking a deep dive into the practicalities of manufacturing a reproducible cell therapy can be sobering. Biomanufacturing is a mature industry, and cell therapy sponsors have an opportunity to learn a significant amount from the successful production of biomaterials and biomolecules; however, the equipment and processes being used today are optimized to remove and produce aqueous components, not the cells themselves. Equipment manufacturers and cell therapy developers are attempting to modify these techniques to isolate, grow, and safely harvest cells, yet perfect solutions do not yet exist. Further, cell therapy production methods are often complex, initially performed by highly trained scientists and engineers, and are challenging to simplify into robust industrial processes that are required for commercialization. Because these challenges are coupled with variability in both incoming tissue and raw materials, the barriers to successful commercial manufacturing of a cell therapy are high. Furthermore, assays and quality standards to evaluate cell potency and viability are not well defined yet and in many cases considered trade secret.

Currently, DiscGenics, Inc. is developing an allogeneic, homologous cell therapy comprised of biomedically engineered progenitor cells derived from donated adult human intervertebral disc tissue to treat chronic low back pain caused by degenerative disc disease. Having successfully completed preclinical studies demonstrating promising bioactivity and consistent safety in animal models and in vitro evaluations, clinical evaluations of this novel product are ongoing (clinicaltrials.gov identifier NCT03347708 and NCT03955315). We are transitioning from bench scale production methods to industrial scale cGMP manufacturing techniques. During this scale-up process, we have identified several sources of variability that have the potential to introduce unacceptable levels of variability into the process, which must be actively mitigated.

In addition to variability challenges in manufacturing, we also face clinical challenges associated with variability, specifically the lack of rigorous diagnostic criteria to establish that a specific degenerated disc is the cause of a patient's axial low back pain. Axial back pain due to degenerative disc disease is the first indication for our first product, Injectable Disc Cell Therapy (IDCT). The treatment is a single, intradiscal injection of live cells intended to reduce pain and disability, through both regenerative and anti-inflammatory mechanisms. Back pain can occur for a number of reasons (genetics, injury, etc.) and can have complicating factors that make identification of mechanical pain challenging [3]. Such pain must also be separated from pain being generated by the facet joints, sacral iliac joints, the low back musculature, or disc herniation, which would not likely be resolved by this type of injection. Identifying patients who would appropriately respond to the treatment in a manner likely to show significance in a prospective, randomized, double-blinded, and controlled clinical study is critical, yet widespread standardization does not exist for such diagnoses related to low back pain. As a result, we took steps to identify ways to decrease variability in our patient population through extensive inclusion/exclusion criteria, as well as minimizing executional issues that can cloud study results.

In this review, we will discuss the regulatory environment for cell therapies in the USA and then highlight key areas of potential variability in both manufacturing and clinical testing.

\section{Regulatory Pathways for Cell Therapies in USA}

FDA's Center for Biologics Evaluation and Research (CBER) regulates "Human Cells, Tissues and other cellular and tissuebased Products" or "HCT/Ps" through two different pathways described in 21 CFR Part 1271 which reflects what the FDA considers to be relative risk. These pathways are for products known as 351 and $361 \mathrm{HCT} / \mathrm{Ps}$ (Fig. 1), so designated because they are described in Section 351 or 361 of the Public Health Service Act (PHSA). Also, cell therapies are generally categorized into autologous (from the patient for the patient) and allogeneic (from a donor for multiple recipients) categories.

The FDA regulates products under Section 361 if they meet very specific criteria outlined in 21 CFR 1271.10(a). These products do not require premarket review or approval by the FDA, as regulation of these products primarily concerns product safety, and more specifically, to "prevent the introduction, transmission or spread of communicable diseases" [4]. While 361 products do not require licensure, the product's manufacturer must adhere to certain manufacturing standards to ensure that appropriate donor eligibility measures are in place and current Good Tissue Practices (cGTP) are being followed.

Products that do not meet the criteria in 21 CFR 1271.10(a) are regulated under Section 351 of the PHSA. In addition to meeting a series of premarket review and approval requirements, including execution of clinical studies under an investigational new drug allowance (IND) to demonstrate the product's safety and efficacy, manufactured cell therapy developers must comply with current good manufacturing (cGMP) regulations for drug and biological products (21 CFR 210/211) issued by FDA.

\section{$361 \mathrm{HCT} / \mathrm{Ps}$}

The criteria for determining if $361 \mathrm{HCT} / \mathrm{P}$ regulates a product are outlined in FDA's Code of Federal Regulations. The first requirement is that the cells are minimally manipulated, which 


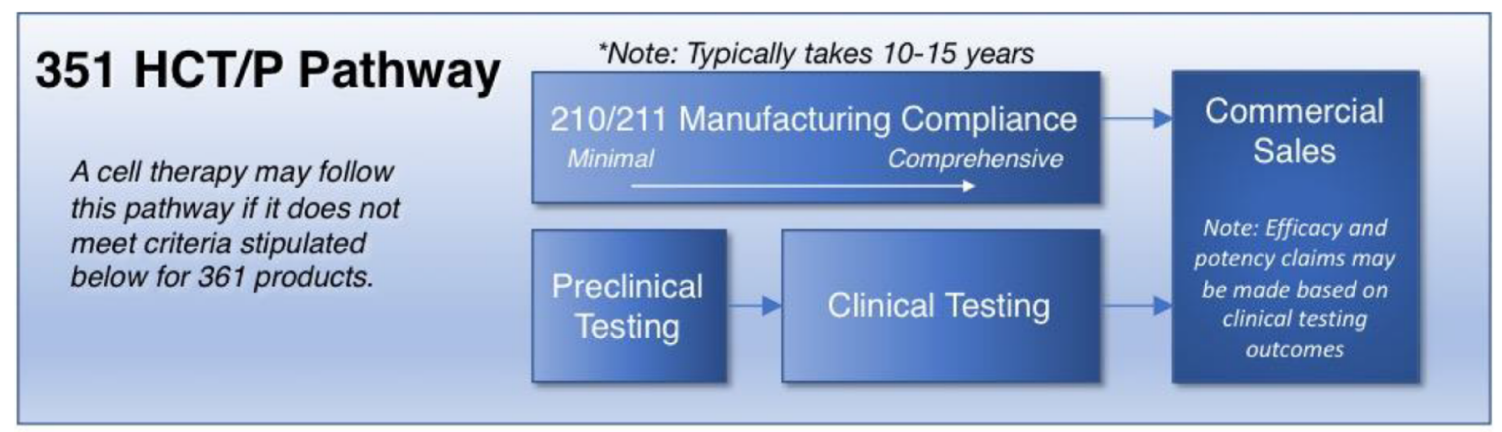

\section{HCT/P Pathway}

A cell therapy may follow this pathway if:

1. Cells are minimally manipulated

2. Intended for homologous use

3. Cells not combined with other materials

4. Only rely on metabolic activity of cells if autologous

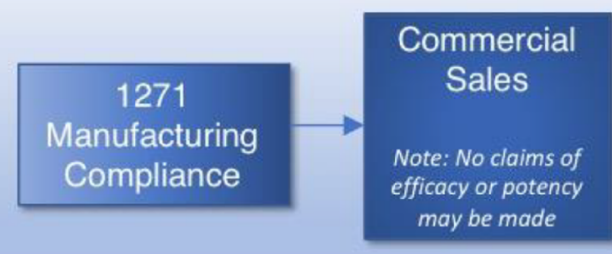

Fig. 1 Two regulatory pathways for cell therapy products, 351 vs 361

commonly means that the cells are not changed, expanded, or differentiated/dedifferentiated in a flask, beaker, or other vessel. They also cannot be exposed to agents, such as media, that change gene expression or function. FDA defines this as "processing that does not alter the relevant biological characteristic of cells" [5].

The next criterion is that cells must only be intended for homologous use, meaning that the cells must continue to be used for their original biological purpose or in their original biological location. Additionally, cells may not be combined with any other material (except water, crystalloids, or storage agent). Finally, the product may only rely on the metabolic activity of the living cells as a primary function if the cells are used autologously (from the same patient).

Manufacturers of products that meet the criteria for categorization as a $361 \mathrm{HCT} / \mathrm{P}$ are responsible for complying with the manufacturing standards outlined in 21 CFR 1271. These include, but are not limited to, registering the facility with the FDA and complying with any audit requests, establishing procedures to determine donor eligibility to minimize disease transmission, and adhering to cGTP for manufacturing. These standards include facility controls and personnel training, raw material controls, and methods for equipment cleaning and calibration. The manufacturing process must be validated, and any changes must be documented and controlled within a Quality System. Testing should be used to understand, and subsequently minimize, the risk of transmission of communicable diseases or other identified safety concerns, such as endotoxin or particulates. Labeling, storage, and records must also be controlled. If a manufacturing deviation occurs, an investigation must be performed.
Importantly, no efficacy or potency claims may be made for such products, given a lack of rigorous clinical testing and lot release potency testing that is intended to ensure consistency of the cell therapy product from lot to lot.

\section{$351 \mathrm{HCT} / \mathrm{Ps}$}

The 351 cell therapy regulatory pathway closely resembles that of a traditional pharmaceutical product, with timelines to market from 10 to 15 years. Products that meet the criteria for categorization as a $351 \mathrm{HCT} / \mathrm{P}$ require evaluation in clinical trials executed in partnership with the FDA. Prior to clinical testing, extensive preclinical evaluation is necessary. The preclinical testing includes studies to understand the product characteristics, numerous in vivo proof of concept studies which explore safety and mechanism of action, and pivotal animal studies which adhere to Good Laboratory Practices (GLP). The sponsor must demonstrate that the potential benefit of the treatment outweighs any potential safety risks found in the in vitro and in vivo preclinical testing. Often, multiple rounds of meetings with the regulators are required to sufficiently answer all safety, manufacturing, and quality questions prior to being granted allowance to initiate clinical trials.

Next, clinical testing must be executed in multiple stages to first understand the safety profile, and subsequently the efficacy of the product in comparison to a clinical control or current standard of care. The use of more than a single clinical site as well as utilizing double-blinded treatment groups can significantly reduce bias during clinical evaluation. Both clinical protocols and final clinical reports are reviewed in detail with the FDA, which may ultimately lead to an approval for 
commercial use whereby a label is designated based upon the efficacy demonstrated in the clinical trials. Such clinical trials can take many years to complete and analyze.

In addition to preclinical testing and clinical testing, manufacturing of the product must adhere to 21 CFR 210/ 211 , and other applicable standards. The product is also subject to identity and potency testing that is correlated with clinical efficacy. The manufacturing site is audited by FDA prior to licensure for commercial use. Because the product characteristics of a cell therapy are tied with clinical efficacy, significantly more manufacturing controls and analytical methods are utilized in 351 products to ensure that a consistent product is delivered to patients. Mirroring the biomanufacturing field, extensive process characterization and validation are employed, along with the use of validated analytical methods, to ensure that a quality product is generated reproducibly. All manufacturing information must be shared in a transparent manner with FDA to ensure complete adherence to regulations. Importantly, products that have established clear safety and efficacy through FDA-allowed clinical trials may make efficacy and potency claims when marketing these therapies to the public.

Regulating cell therapies is a complex task for the US FDA and other regulators around the world. In the USA, the onus is on manufacturers to evaluate criteria outlined by the FDA to determine the most appropriate regulatory pathway for their product. However, as science and technology develop, novel cell therapies do not always fit squarely into the two regulatory pathways. Recent actions by the FDA suggest that some companies are inaccurately self-designating their products as $361 \mathrm{HCT} / \mathrm{Ps}$ and marketing manufactured cell therapies without taking the necessary steps to ensure patient safety and product characterization. Recently, the FDA has taken action to clarify regulations and ensure that all manufacturers understand and adhere to the appropriate quality and safety standards. The agency has issued a number of new guidance documents as well as taken legal action against inaccurately selfdesignated organizations and is more strongly enforcing the requirement of cGMP manufacturing controls and clinical trial evidence.

\section{Sources of Variability in Manufacturing}

For the purposes of this discussion around sources of variability in manufacturing, a simplified manufacturing process of a 351-type allogeneic cell therapy is proposed (Fig. 2). First, biologic raw material containing cells derived from humans is obtained. Next, the cells are isolated and expanded, resulting in some desired phenotypic change. Finally, the cells are harvested and frozen. After lot release testing, the product is transported to the clinical site, thawed and prepared according to instructions and applied to the patient.
At each point in this manufacturing process, there are several factors unique to cell therapy manufacturing that can contribute to adding variability to the final product. Identifying these factors, understanding the range of variability, and subsequently using techniques to minimize variability, ultimately leads to a more consistent final product and fewer lots failing final lot release.

A Quality by Design (QbD) approach is used to develop and describe this manufacturing process [6]. The key tenet of $\mathrm{QbD}$ is that quality cannot be tested into products but should be built in by design. The QbD effort entails a series of risk assessments, characterization studies, as well as the validation of identity, potency, and safety metrics. Adherence to a welldesigned QbD strategy results in the determination of critical quality attributes and process parameters as they relate to an overall quality target product profile.

Process variability is understood and minimized through a process known as characterization. In this effort, all process parameters and attributes are evaluated for their association and impact. Design of experiment (DOE) is a useful tool in executing such characterization for a biomanufacturing process. DOE in this context is used to systematically cover the entire mathematical space, resulting in reliable quantification of cause, effect, two-way interactions, quadratic responses, and random variation for high-risk inputs. In the context of cell therapy, critical quality attributes measure the linkage of measurable molecular and cellular characteristics of a cell population to final product quality.

\section{Biological Raw Materials}

All cellular therapies begin with an initial cell source from a donor or from a cell line; unfortunately, donor-specific cells are particularly prone to introducing variability into the manufacturing process. Qualification and characterization studies of the starting biological raw material must be performed in order to understand the properties of the starting cells to ensure they can yield the desired final cell output. These activities include determination of donor eligibility with respect to safety (risk for adventitious agent transmission must be mitigated), evaluation of patient characteristics (age, gender, medical history, etc.), and analytical evaluation of tissue and cell properties (analysis of gene expression, functionality, quality and amount of cells/tissue, etc.). Regulations do not allow for cells to be combined from different donors into a single lot [4]. Because donors cannot be combined, variability exists in the starting amount of tissue harvested and expanded. To minimize biologic raw material variability, both in terms of quantity and quality, deep knowledge of the properties of the incoming material coupled with validated analytical data shown to discern appropriate properties of the cells must be utilized. Extensive donor characterization must be performed 


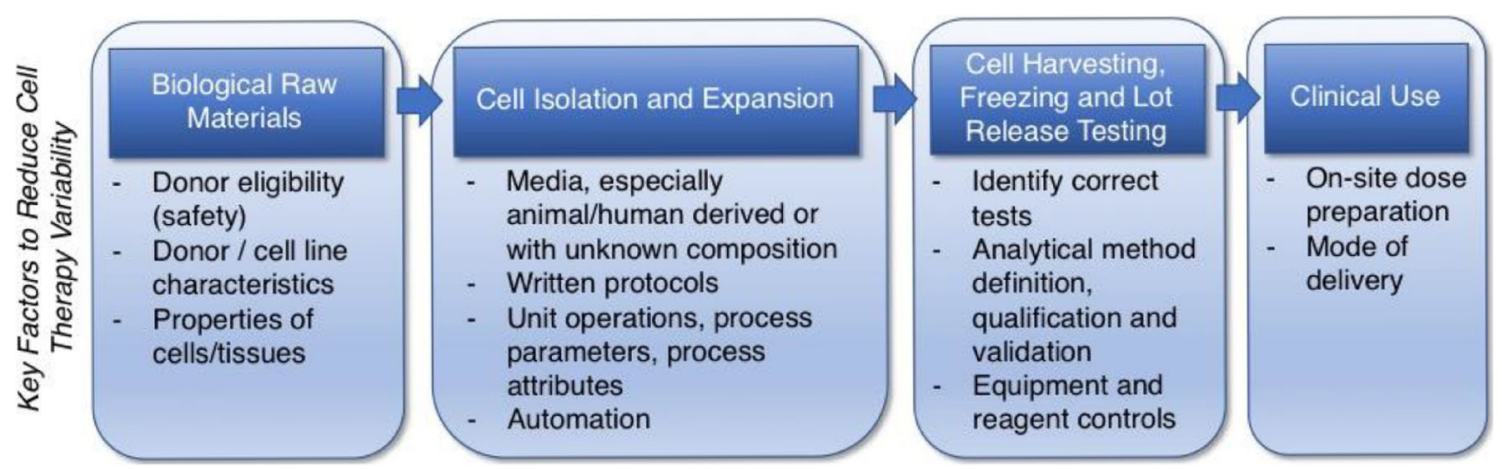

Fig. 2 A hypothetical allogeneic cell therapy process and key aspects to consider in order to minimize product variability

(on the order of dozens to hundreds of lots) to identify true trends in the dataset, depending on the variability seen.

\section{Cell Isolation and Expansion}

During the cell isolation and expansion phase, there are a number of aspects that can contribute to heightened and potentially unacceptable levels of variability in the final product. A major consideration is the media used during the process. These reagents, which drive cell growth and behavior, must be carefully risk assessed, understood, and controlled in a manner commensurate with the risk they pose to the process. Of heightened concern are materials with compositions that are not disclosed by the vendors and undefined raw materials that contain undefined human or animal-derived components. Due to the impact of raw materials on stem cell differentiation, fetal bovine serum is a particularly challenging reagent to replace for cell therapies. The desire to move away from bovine reagents due to disease transmission concerns has spurred research for using human platelet lysate as a substitute, but this reagent can lead to unacceptable changes in cell phenotype and potency, and also can introduce even more variability due to its own manufacturing challenges [7]. Despite repeated attempts to replace serum, a suitable alternative remains elusive [8].

Once a media composition is selected, it must be thoroughly understood and controlled through qualification activities. Media qualification can include replicating some or all aspects of the Certificate of Analysis, as well as performing custom tests, such as growth or differentiation assays from multiple lots of media, on a periodic basis, and from each vendor. Cell therapy manufacturers should test all incoming raw materials using techniques such as FTIR or HPLC to ensure that the proper material is being provided by the vendor.

Another source of variability during this phase of manufacturing has to do with lack of consistency within the process itself. As previously mentioned, these processes are often complex and require many steps, which can vary by operator and by day. Early stage protocols lack the step-by- step definition necessary to eliminate subjective operator decision making, which inadvertently introduces variability into the final product. For scientific rigor and manufacturing purposes, extremely detailed protocols that explicitly define every step must be generated. During this documentation process, identifying clear unit operations with specified ranges for process parameters and attributes improves transparency of the process, facilitates risk assessments, and enables robust process characterization.

Along with thorough documentation, opportunities to automate steps, especially those that require user input or subjective decision making, should be considered. For example, assessment of confluency in adherent culture can vary from person to person. To mitigate operator-driven risk, various custom microscopes and software have been developed to quantitatively determine the appropriate harvest time. While the hemocytometer is the gold standard for counting cells, more advanced technology that automates the sample preparation and analysis is now available that can significantly reduce variability.

\section{Cell Harvesting, Freezing, and Lot Release Testing}

When the culturing process is complete, cells are harvested, and in many cases, frozen to $-196{ }^{\circ} \mathrm{C}$ in the vapor phase of liquid nitrogen. At this point, stability can be maintained for many years, allowing for both rigorous lot release testing and a long shelf life prior to clinical use. Analytical methods are chosen by the manufacturer (and discussed with regulators) to elucidate the purity, potency, identity, and safety of the cell product. The potency assays should reflect the proposed mechanism of action and tie to the clinical activity noted in human testing. When possible, assays should meet compendial standards, which simplifies how FDA scrutinizes the assay and can also alleviate some of the validation activities.

Analytical methods must be defined with a detailed protocol, qualified, and finally validated to demonstrate properties, such as linearity, precision, and accuracy. Additionally, the 
equipment and reagents used within the methods must also be defined and controlled using the validated techniques previously described. Only when these assays are properly established can process variability be truly understood; therefore, the analytical team of a cell therapy company must be well-resourced and supported, since they hold the key to demonstrating process consistency. Some of the release assays used in cell therapy can pose particular challenges for validation due to the complexity of the methods, such as the use of biological assays. Further, while a few tests may suffice for a traditional small molecule or biologic, cell therapies often require dozens of release tests to address the complex mechanisms of action and various safety concerns that may be present within a manufactured cell therapy, which can make the task of establishing these assays a significant one.

\section{Clinical Use}

Once the dose meets the preset criteria for lot release, it can be delivered to the clinical site. Unfortunately, while the manufacturer may go to great lengths to reduce variability in the product during the manufacturing process, clinical implementation of the product may introduce new variability concerns. Cell therapies often require sophisticated supply chain and handling. Also, they require some level of manipulation prior to use, which can range from simply thawing the vial to more complex manipulations such as mixing or centrifugation. These extra steps can introduce potential variability, so developing a simple process that considers the skills and resources available at the clinical site is paramount. Exhaustive training and the implementation of stop-gates help to ensure that a viable and non-contaminated product is delivered to the patient. In general, the hope is that as the cell therapy field matures, technologies and processes will evolve such that clinical site manipulations become minimal if not completely eliminated.

Along with dose preparation, the mode of delivery must also be highly controlled. Live cells can be sensitive to shear forces, shifts in temperature or other factors that can compromise the viability, and therefore potency, of the product. In many cases, cell therapies treat novel indications where standard delivery methods are not yet established, so development of new delivery techniques and training for the treating physicians is necessary.

\section{Sources of Variability in Clinical Trials}

Successful execution of clinical trials relies upon including patients within the study who are likely to respond in a similar manner to the treatment provided. In this way, by randomizing control groups into a study, true efficacy can be determined. In some medical conditions, patient identification is straight forward, for example through a blood test or definitive imaging criterion. In the case of low back pain, which is the target indication for DiscGenics' allogeneic cell therapy, diagnosis and treatment modalities vary from clinic to clinic. Uniquely, patients who experience low back pain may not actually show radiographic evidence of degeneration, and conversely, patients with radiographic evidence of disc damage may not experience pain. Clinical observations, interventional assessment techniques, such as discography, and radiographic imaging must be considered when determining a diagnosis of symptomatic degenerative disc disease [9]. The research community is racing to better understand the etiology of the disease in order to more appropriately differentiate these patients; however, in the meantime, clinicians must find ways to identify these patients through multiple diagnostic and radiographic tools, and sorting through symptoms to find the appropriate treatment [10].

When the medical community lacks a clear standard for patient identification, efforts must be made to identify and facilitate discussions among key opinion leaders (physicians and other expert clinicians) that are necessary to identify inclusionary and exclusionary criteria for patient selection. Production of a robust clinical protocol written in collaboration with key opinion leaders (KOLs) is the cornerstone for optimizing the design of the clinical study. Many protocols fail in the real word setting due to lack of consultation with actively working physicians. Regulators should also be included in the discussion, as they often have access to unpublished studies that can help aid in study design and define appropriate patient enrollment criteria, thereby improving the likelihood of a successful study. Ultimately, regulators will determine eligibility of $351 \mathrm{HCT} / \mathrm{Ps}$ for licensure, and improper patient selection that results in too diverse of a patient population can be a cause of licensure denial.

Having complex inclusion and exclusion criteria can be challenging to manage, as clinical sites may find it onerous to sift through and reject a large percentage of potential patients. However, despite the longer enrollment periods, such discipline is key to improving the likelihood of clinical trial success. To start with a sufficiently high number of patients, great efforts must be taken to advertise and recruit potential patients to the clinical sites for pre-screening, screening, and potential study enrollment (Fig. 3).

Clinical site variability cannot be avoided in multi-center studies but is manageable through stringent site feasibility evaluation, selection, and extensive start-up processes that includes training. Site feasibility evaluation is critical to ensuring that each clinical site has the appropriate experience and qualifications. Also, hosting Investigator Meetings where the clinicians, nurses, and staff executing the clinical trial review the clinical protocol, logistics, and documentation plans can limit miscommunication and improve trial success. 
Fig. 3 Potential patients must be screened through rigorous inclusion and exclusion criteria prior to being admitted into the clinical study, even though the percentage allowed may be small relative to those seeking treatment

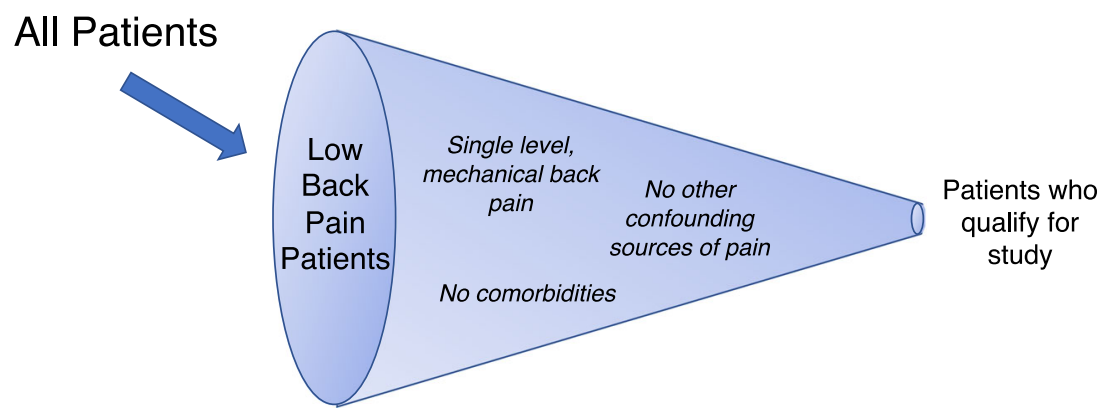

Once the correct patients are identified, adherence to Good Clinical Practices (GCPs) and implementation of protocols, changes via amendments, and electronic data capture systems that are monitored by trained Clinical Research Associates (CRAs), among other steps, enhance the possibility of smooth trial execution and minimize variability caused by human error or miscommunication. When subjective evaluations are involved, the use of central labs with consistently trained professionals utilizing pre-set parameters for analysis is key in minimizing site-by-site bias. Similarly, evaluating patient outcomes in a blinded manner can remove potential bias that introduces variability into the dataset. Other simple ways to prevent assessment bias include providing clinical site staff with scripts and hard copies of measured assessments that have been approved by the relevant Institutional Review Boards (IRBs) to ensure consistency and avoid misinterpretation, and utilizing the same imaging equipment for each patient.

\section{Conclusions}

The field of cell therapy is experiencing rapid growth, with a convergence of regulatory approvals, enabling manufacturing technology improvements, and a strong funding environment. Many promising treatments in the pipeline are positioned to change the way medicine is practiced. However, manufacturers face large hurdles before commercialization can be realized. While regulators are actively updating guidance documents and aligning the way they respond to inquiries, the speed of cell therapy development is moving quickly and, at times, outpaces regulations. Ultimately, while we strive to provide a consistent and efficacious product, patient safety is the primary concern, and compliance with the appropriate regulatory standards supports that mission.

While all cell therapies face issues related to media variability and processing controls, allogeneic cell therapies that start from donor material face inherently more variability challenges than cell therapies derived from a single cell source. Employing a Quality by Design paradigm for process definition, validation, and continual improvement facilitates identifying and minimizing manufacturing variability risks, resulting in a more consistent and well-understood process.
Given the high value of cell therapy products, there is little tolerance for failed lots, and therefore exhaustive process characterization and optimization are essential in this field.

In the clinic, reducing variability in treatment preparation and delivery, as well as identifying a homogeneous patient population, is critical to improving the likelihood of seeing clinical benefit of a potential new therapy in a trial. By designing prospective, randomized, double-blinded, placebocontrolled clinical trials using a high-quality GMP investigational product, we increase the likelihood that these promising treatments gain market approval and have the ability to help the millions of patients suffering without current recourse. As an industry, setting a high bar for manufacturing and clinical execution will expedite approvals and foster trust from the regulatory, medical, and patient communities for cellular therapies.

Funding Information This material is based in part upon work supported by the National Science Foundation Graduate Research Fellowship under Grant No. 1451511. Any opinion, findings, and conclusions or recommendations expressed in this material are those of the authors and do not necessarily reflect the views of the National Science Foundation.

\section{Compliance with Ethical Standards}

Conflict of Interest L Silverman, F Flanagan, D Rodriguez-Granrose, and K Simpson are employees at DiscGenics. L Silverman, F Flanagan, D Rodriguez-Granrose, K Simpson, L Saxon, and K Foley own stock or stock options in DiscGenics. L Saxon is an independent consultant to DiscGenics. F Flanagan and K Foley are on the board of DiscGenics. F Flanagan is on the board of the Alliance for Regenerative Medicine.

Open Access This article is distributed under the terms of the Creative Commons Attribution 4.0 International License (http:// creativecommons.org/licenses/by/4.0/), which permits unrestricted use, distribution, and reproduction in any medium, provided you give appropriate credit to the original author(s) and the source, provide a link to the Creative Commons license, and indicate if changes were made.

\section{References}

1. FDA. https://www.fda.gov/vaccines-blood-biologics/cellular-genetherapy-products/approved-cellular-and-gene-therapy-products. Accessed July 24, 2019. 
2. Q1 2019 Data report Alliance for regenerative medicine. 2019. https://alliancerm.org/publication/q1-2019-data-report/. Accessed July 172019.

3. Dagenais S, Tricco AC, Haldeman S. Synthesis of recommendations for the assessment and management of low back pain from recent clinical practice guidelines. Spine J. 2010;10(6):514-29.

4. Guidance for industry: current good tissue practice (CGTP) and Additional Requirements for Manufacturers of Human Cells, Tissues, and Cellular and Tissue-Based Products (HCT/Ps). US Department of Health and Human Services Food and drug Administration Center for Biologics Evaluation and Research Center for devices and radiological health Office of Combination Products. December 2011; https://www.fda.gov/media/82724/ download. Accessed 17 July 2019.

5. Regulatory considerations for human cells, tissues, and cellular and tissue- based products: minimal manipulation and homologous use, Guidance for industry and Food and Drug Administration staff. US Department of Health and Human Services Food and drug Administration Center for Biologics Evaluation and Research Center for devices and radiological health Office of Combination Products. December 2017; https://www.fda.gov/media/109176/ download. Accessed 17 July 2019.
6. Lipsitz YY, Timmins NE, Zandstra PW. Quality cell therapy manufacturing by design. Nat Biotechnol. 2016;34(4):393-400.

7. Burnouf T, Strunk D, Koh MB, Schallmoser K. Human platelet lysate: replacing fetal bovine serum as a gold standard for human cell propagation? Biomaterials. 2016;76:371-87.

8. Karnieli O, Friedner OM, Allickson JG, Zhang N, Jung S, Fiorentini D, et al. A consensus introduction to serum replacements and serum-free media for cellular therapies. Cytotherapy. 2017;19(2):155-69.

9. H Serhan. Advancements in the treatment of degenerative disc disease. Hamdan Med J 2018;11:175-183.

10. Taher F, Essig D, Lebl DR, et al. Lumbar degenerative disc disease: current and future concepts of diagnosis and management. Adv Orthop. 2012;2012:970752.

Publisher's Note Springer Nature remains neutral with regard to jurisdictional claims in published maps and institutional affiliations. 